PROCEEDINGS OF THE

AMERICAN MATHEMATICAL SOCIETY

Volume 42, Number 1, January 1974

\title{
THE DENSITY CHARACTER OF FUNCTION SPACES
}

\author{
N. NOBLE
}

\begin{abstract}
For topologies between the pointwise topology and the compact-open topology, the density character of $C(X)$ (and, for certain spaces $Z, C(X, Z)$ ) is described in terms of a cardinal invariant of $X$. The Hewitt-Pondiczery theorem on the density character of product spaces follows as a corollary.
\end{abstract}

1. Description. Except in Corollary 2, all hypothesized spaces are assumed to be completely regular Hausdorff. The set of continuous functions from $X$ to $Z$ is denoted by $C(X, Z)$ or, when $Z=R$, by $C(X)$. The density character, $\delta X$, of a space $X$ is the least cardinality of a dense subset of $X$ and the weight, $w X$, of $X$ is the least cardinality of an open basis of $X$. We define the weak weight, wwX, of $X$ to be the least of the cardinals $w Y$ for $Y$ a continuous one-to-one image of $X$.

THEOREM 1. Let $X$ be any infinite space and let $C(X)$ have any topology between the pointwise topology and the compact-open topology. Then $\delta C(X)=w w X$.

All proofs will be given in the next section. Our remaining results allow Theorem 1 to be applied to yield information about $\delta C(X, Z)$ for suitable paces $Z$.

LEMmA. Let $C(X, Z)$ and $C\left(X, Z^{*}\right)$ both have either the topology of uniform convergence on members of some fixed cover of $X$ or the set-open topology generated by such a cover. If $Z$ is a retract of $Z^{*}$ then $\delta C(X, Z) \leqq$ $\delta C\left(X, Z^{*}\right)$.

Proposition. For any topologies between the pointwise topology and the compact-open topology:

(i) For any space $Z, \delta Z \leqq \delta C(X, Z)$.

(ii) If $Z$ contains a nondegenerate arc, then $\delta C(X) \leqq \delta C(X, Z)$.

(iii) If for each finite subset $F$ of $X$ there exists a function $f$ in $C(X, Z)$ such that $F$ and $f(F)$ have the same cardinality, then $\delta C(X) \leqq \delta C(X, Z) \cdot$ $\delta C(Z)$.

Received by the editors February 26, 1971.

AMS (MOS) subject classifications (1970). Primary 46E10, 54C35; Secondary 54D10.

Key words and phrases. Density character, separable spaces, function spaces.

(c) American Mathematical Society 1974 
THEOREM 2. Suppose $Z$ contains more than one point and is a retract of a convex subset of some locally convex linear space. For any topologies between the pointwise topology and the compact-open topology, $\delta C(X, Z)=$ $\delta C(X) \cdot \delta Z$.

CoROllaRY 1. For $Z$ as in Theorem 2 and any topology between the pointwise topology and the compact-open topology, $C(X, Z)$ is separable if and only if $Z$ is separable and some continuous one-to-one image of $X$ is separable metrizable.

Under the same hypotheses on a separable space $Z$, Vidossich [6] shows that $C(X, Z)$ is separable if and only if $X$ is submetrizable (has a continuous one-to-one metrizable image) with $\delta X \leqq c$, which is equivalent to being submetrizable and of cardinality less than or equal to $c$. Combined with Corollary 1 this yields:

COROLlary 2. On a set of cardinality less than or equal to $c$ each metrizable topology contains a separable metrizable topology.

THEOREM 3. For the topology of pointwise convergence, if $Z$ is arcwise connected or if $X$ is a P-space then $\delta C(X, Z) \leqq \delta C(X) \cdot \delta Z$.

Corollary 3 (Hewitt [2], Pondiczery [3]). Let $\mathrm{n}$ be an infinite cardinal. With no separation axioms assumed, let $X=\prod_{\alpha \in A} X_{\alpha}$ where each $X_{\alpha}$ has $\delta X_{\alpha} \leqq n$. If card $A \leqq 2^{\mathfrak{n}}$, then $\delta X \leqq n$.

Turning to other topologies on $C(X)$ we note that the compact-open topology arises in Theorem 1 only because we need the Stone-Weierstrass Theorem (that a subalgebra is dense if it contains the constants and separates points). When dealing with a subalgebra generated by a set of cardinality $n$ which separates points, compactness can be weakened to weak- $n-\boldsymbol{\kappa}_{0}$-compactness (each $n$-fold open cover has a finite subcollection whose union is dense-such spaces are studied, for example, in [4]) so if $w w X=n$, then in the set-open topology generated by all weakly- $n-\kappa_{0}$ compact subsets of $X, \delta C(X) \leqq n$. In case $w w X=\aleph_{0}$, an even stronger statement holds: $C(X)$ is separable in the set-open topology generated by all relatively pseudocompact subsets of $X$. (A set $S \subseteq X$ is relatively pseudocompact if each function in $C(X)$ is bounded on $S$; weak- $\boldsymbol{\aleph}_{0}-\boldsymbol{K}_{0}$ compactness is equivalent to pseudocompactness.)

Of course the most interesting topology on $C(X)$ larger than the compact-open topology is the topology of uniform convergence, and for this topology it is known that $\delta C(X)=w \beta X$ : That $\delta C^{*}(X)=w \beta X$ is established by Smirnov in [5] by a proof similar to the proof of Theorem 1 and the more difficult result $\delta C^{*}(X)=\delta C(X)$ is shown by Comfort and Hager in [1]. 
Since the largest set-open topology on $C(X)$ is the topology of uniform convergence if (and only if) $X$ is pseudocompact, it follows by the Comfort-Hager-Smirnov Theorem, the comments above, and the fact that $\beta X$ is separable only if $X$ is compact, that if $X$ is compact separable metric, no strictly larger topology on $X$ is pseudocompact.

2. The proofs.

Proof of Theorem 1. We first show that $\delta C(X) \leqq w w X$; since larger topologies have "larger" density characters, it suffices to consider the case where $C(X)$ has the compact-open topology. Let $\mathscr{B}$ be a base for some topology contained in the topology of $X$ with card $(\mathscr{B})=w w X$ and form a set of functions $D \subseteq C(X, I)$ by choosing, for each pair $(U, V)$ in $\mathscr{B} \times \mathscr{B}$ with $U \subseteq V$, a function which maps $U$ to 0 and $X \backslash V$ to 1 , if such a function exists. Clearly $D$ separates points of $X$, so by the StoneWeierstrass Theorem (for example, [7, problem 44B]) the algebra generated by $D$ and the constant functions is dense in $C(X)$. Hence the set, $D^{*}$, of all finite linear combinations, with rational coefficients, of members of $D$ or rational constant functions is dense in $C(X)$. Since card $D^{*}=$ card $D=w w X$, this shows that $\delta C(X) \leqq w w X$.

For the remaining relation, $w w X \leqq \delta C(X)$, it suffices to consider $C(X)$ in the pointwise topology. Let $D \subseteq C(X)$ be dense with card $(D)=\delta C(X)$ and let $e: X \rightarrow R^{D}$ be the embedding map, $e(x)_{d}=d(x)$. Since $D$ is dense, it separates points, so $e$ is one-to-one. Since each subspace of $R^{D}$ has weight $\leqq$ card $D=\delta C(X)$, it follows that $w w X \leqq \delta C(X)$.

ProOF OF the lemma. For $D \subseteq C\left(X, Z^{*}\right)$ dense and $r: Z^{*} \rightarrow Z$ a retraction, $\{r \circ d: d \in D\}$ is dense in $C(X, Z)$.

PROOF OF THE PROPOSITION. (i) Identifying $Z$ with the constant functions and choosing $z$ in $Z$, the function which carries $f$ to $f(z)$ is a retraction of $C(X, Z)$ to $Z$. Thus, by the lemma, $\delta Z \leqq \delta C(X, Z)$.

(ii) By the lemma $\delta C(X, I) \leqq \delta C(X, Z)$ and since

$$
C^{*}(X)=\bigcup_{n} C(X,[-n, n])
$$

is dense in $C(X), \delta C(X)=\delta C(X, I)$. Therefore $\delta C(X) \leqq \delta C(X, Z)$.

(iii) To see that $\delta C(X) \leqq \delta C(X, Z) \cdot \delta C(Z)$ it suffices to consider the pointwise topologies, since by Theorem $1, \delta C(X)$ does not change, and $\delta C(X, Z)$ can only be larger. Let $D_{1}$ and $D_{2}$ be dense in $C(X, Z)$ and $C(Z)$ respectively with card $D_{1}=\delta C(X, Z)$, card $D_{2}=\delta C(Z)$, and set $D=$ $\left\{g \circ f: f \in D_{1}, g \in D_{2}\right\}$. We show that $D$ is dense in $C(X)$. Given distinct points $x_{1}, \cdots, x_{n}$ in $X$ and open subsets $U_{1}, \cdots, U_{n}$ of $R$ with $\bigcap_{i=1}^{n} N\left(x_{i}, U_{i}\right) \neq \varnothing$ (where $N(x, U)=\{f: f(x) \in U\}$ ), there exists a function $h$ in $C(X, Z)$ such that the points $h\left(x_{i}\right)$ are all distinct. Choosing disjoint open neighborhoods $V_{i}$ of $h\left(x_{i}\right), \bigcap_{i=1}^{n} N\left(x_{i}, V_{i}\right)$ is thus a nonempty open 
set, so it contains a function $f$ in $D_{1}$. Since $\bigcap_{i=1}^{n} N\left(x_{i}, U_{i}\right) \neq \varnothing$, it contains a function which maps the points $x_{i}$ to distinct reals and hence, shrinking the $U_{i}$ if necessary, we may suppose that they are all disjoint. Thus $\bigcap_{i=1}^{n} N\left(f\left(x_{i}\right), U_{i}\right)$ is not empty, so it contains a function $g$ in $D_{2}$. But now $g \circ f$ is in $\bigcap_{i=1}^{n} N\left(x_{i}, U_{i}\right)$, as desired.

Proof of TheOREM 2 . Since $Z$ must contain a nondegenerate arc, the relation $\delta C(X, Z) \geqq \delta C(X) \circ \delta Z$ follows from the first two parts of the proposition. For the reverse inequality it suffices to consider the case where $Z$ is a convex subset of a locally convex linear space $Z^{*}$ which contains the origin and where $C(X, Z)$ and $C(X)$ bear the compact-open topologies. Let $D^{\prime}$ be a dense subset of $C(X, I)$ of cardinality $\delta C(X)$ (that $\delta C(X, I)=\delta C(X)$ follows from the lemma and the second part of the proposition) and let $D^{\prime \prime}$ be the set of convex combinations, with rational coefficients, of members of $D^{\prime}$. For $g$ and $g^{\prime}$ in $D^{\prime \prime}$ and for $r$ and $s$ rational with $0 \leqq r<s \leqq 1$ choose $h\left(g, g^{\prime}, r, s\right)$ in $C(X, I)$ such that for $h=$ $h\left(g, g^{\prime}, r, s\right), h(x) \leqq g^{\prime}(x)$ for all $x$ in $X, h(x)=g^{\prime}(x)$ if $x$ is in $g^{-1}([0, r])$, and $h(x)=0$ if $x$ is in $g^{-1}([s, 1])$. Finally let $D_{1}$ be the set of all functions $h\left(g, g^{\prime}, r, s\right)$ so chosen and note that the cardinality of $D_{1}$ is $\delta C(X)$. Now let $D_{2}$ be a dense subset of $Z$ of cardinality $\delta Z$ and, identifying $z$ in $D_{2}$ with the constant function on $X$ whose value is $z$, set

$$
D=\left\{\sum_{i=1}^{m} f_{i} z_{i}: f_{i} \in D_{1}, z_{i} \in D_{2}\right\}
$$

Since the cardinality of $D$ is $\delta C(X) \cdot \delta Z$, it suffices to show that $D \cap$ $C(X, Z)$ is dense in $C(X, Z)$.

Let $K$ be a compact subset of $X$, let $\rho$ be a continuous seminorm for $Z^{*}$, let $\varepsilon$ be a positive rational less than 1 and choose $f$ in $C(X, Z)$. Let $S=$ $\{z \in Z: \rho(z)<\varepsilon / 2\}$, cover $K$ with the sets $U(x)=f^{-1}(f(x)+S)$ for $f(x)$ in $D_{2}$ and let $U\left(x_{1}\right), \cdots, U\left(x_{n}\right)$ be a finite subcover. Let $\Phi_{1}, \cdots, \Phi_{n}$ be a partition of unity subordinate to this cover, let $M$ be a positive integer greater than each of the numbers $2 n \rho\left(f\left(x_{i}\right)\right), 1 \leqq i \leqq n$, and choose functions $f_{i}$ in $D^{\prime}$ such that for each $x$ in $K, \Phi_{i}(x)-\varepsilon / M<f_{i}(x)<\Phi_{i}(x)-\varepsilon / 2 M$. Set $g=(1 / n) \sum_{i=1}^{n} f_{i}, r=1 / n-\varepsilon / 2 M$ and $s=1 / n$ and let $h_{i}=h\left(g, f_{i}, r, s\right)$, $1 \leqq i \leqq n$. Note that for $x$ in $K$,

$$
g(x)=\left(\frac{1}{n}\right)\left(\sum_{i=1}^{n} f_{i}(x)\right) \leqq\left(\frac{1}{n}\right)\left(\sum_{i=1}^{n} \Phi_{i}(x)-\frac{n \varepsilon}{2 M}\right)=r,
$$

so $h_{i}(x)=f_{i}(x)$. Furthermore, for any $x, \sum_{i=1}^{n} h_{i}(x) \leqq \sum_{i=1}^{n} f_{i}(x)$ and $\sum_{i=1}^{n} h_{i}(x)=0$ if $\sum_{i=1}^{n} f_{i}(x) \geqq 1$, so $\sum_{i=1}^{n} h_{i}(x) \leqq 1$. Therefore the function $h=\sum_{i=1}^{n} h_{i} f\left(x_{i}\right)$ is in $D \cap C(X, Z)$. We complete the proof by showing that 
$\rho(h(x)-f(x))<\varepsilon$ for $x$ in $K$. For such $x$,

$$
\begin{aligned}
\rho(h(x)-f(x)) & =\rho\left(\sum_{i=1}^{n} h_{i}(x) f\left(x_{i}\right)-\sum_{i=1}^{n} \Phi_{i}(x) f(x)\right) \\
& =\rho\left(\sum_{i=1}^{n}\left(h_{i}(x)-\Phi_{i}(x)\right) f\left(x_{i}\right)+\sum_{i=1}^{n} \Phi_{i}(x)\left(f\left(x_{i}\right)-f(x)\right)\right) \\
& \leqq \sum_{i=1}^{n} \rho\left(f\left(x_{i}\right)\right)\left|\Phi_{i}(x)-f_{i}(x)\right|+\left(\sum_{i=1}^{n} \Phi_{i}(x)\right)(\varepsilon / 2) . \\
& \leqq \varepsilon / 2+\varepsilon / 2=\varepsilon .
\end{aligned}
$$

Proof of Theorem 3. We first suppose that $Z$ is arcwise connected. Let $D^{\prime}$ be a dense subset of $Z$ of cardinality $\delta Z$ and for each finite subset $F$ of $D^{\prime}$ choose an arcwise connected subspace $X_{F}$ of $Z$ which contains $F$, is the union of finitely many arcs, and which contains no closed loops. (To construct such a subspace, suppose inductively that $Y_{k}$ has been constructed so as to satisfy these conditions and contain $k$ points of $F$, let $z$ be a point in $F \backslash Y_{k}$, let $f$ be an arc with $f(0)$ in $Y$ and $f(1)=z$ and let $r$ be the largest number such that $f(r)$ is in $Y_{k}$. The space $Y_{k+1}=Y_{k} \cup$ $f([r, 1])$ has the required properties and contains $k+1$ points of $F$.) The space $X_{F}$ is a retract of $R^{2}$, so $\delta C\left(X, X_{F}\right) \subseteq \delta C(X)$ and hence $C\left(X, X_{F}\right)$ contains a dense subset $D_{F}$ of cardinality at most $\delta C(X)$. Set $D=$ $\bigcup\left\{D_{F}: F\right.$ is a finite subset of $\left.D^{\prime}\right\}$ and note that the cardinality of $D$ is at most $\delta C(X) \cdot \delta Z$.

Observe that each $X_{F}$ can be expressed as $h_{F}(I)$ for some continuous function $h_{F}$. Hence if $F^{\prime} \subseteq X$ is finite and $f: F^{\prime} \rightarrow F \subseteq D^{\prime}$ is given, then $f$ has a continuous extension $f^{*}: X \rightarrow X_{F}$. (Indeed, for $g: F^{\prime} \rightarrow I$ with each $g(x)$ in $h_{F}^{-1}(f(x))$ and $g^{*}: X \rightarrow I$ a continuous extension of $g$, the function $f^{*}=h_{F} \circ g^{*}$ is such an extension.) It is now easy to show that $D$ is dense. Given distinct points $x_{1}, \cdots, x_{n}$ of $X$ and nonempty open subsets $U_{1}, \cdots$, $U_{n}$ of $Z$, choose points $z_{i}$ in $D^{\prime} \cap U_{i}$, set $F=\left\{z_{1}, \cdots, z_{n}\right\}$, let $f$ be the function which carries $x_{i}$ to $z_{i}$ and let $f^{*}: X \rightarrow X_{F}$ be a continuous extension of $f$. Then $f^{*}$ is in $\bigcap_{i=1}^{n} N\left(x_{i}, U_{i}\right)$, so $\bigcap_{i=1}^{n} N\left(x_{i}, U_{i}\right)$ is not empty. It follows that $D_{F}$, and hence $D$, meets $\bigcap_{i=1}^{n} N\left(x_{i}, U_{i}\right)$, as desired.

Now suppose that $X$ is a $P$-space. Let $Z^{*}$ be the quotient of $I \times Z$ formed by identifying $\{1\} \times Z$ to a point $v$, choose a point $z_{0}$ in $Z$ and let $q: Z^{*} \rightarrow Z$ be the map which carries $v$ to $z_{0}$ and points $(r, z)$ to $z$. Since $Z^{*}$ is arcwise connected, $C\left(X, Z^{*}\right)$ contains a dense subset $D^{\prime}$ of cardinality at most $\delta C(X) \cdot \delta Z$. Note that if $f: X \rightarrow Z^{*}$ is continuous then $q \circ f$ is continuous since $f^{-1}(v)$, being a closed $G_{\delta}$, is both closed and open. Thus $D=\left\{q \circ f: f \in D^{\prime}\right\}$ is a subset of $C(X, Z)$ of cardinality at most $\delta C(X) \cdot \delta Z$. Since $D$ is clearly dense in the pointwise topology, the proof is complete. 
Proof of Corollary 3. Let $X=\prod_{\alpha \in A} X_{\alpha}$ where card $A=2^{n}$ and where, we may suppose, $A$ is discrete and each $X_{\alpha}$ contains a dense subset $D_{\alpha}$ of cardinality $n$. Let $Z_{\alpha}=Z$, the discrete space of cardinality $n$, and let $D^{\prime}$ be a dense subset of $\prod_{\alpha \in A} Z_{\alpha}=C(A, Z)$ of cardinality at most $\delta C(A)$. $\delta Z=w w A \cdot \delta Z=n \cdot n=n$. (Since $A$ is discrete, $w w A$ is the least cardinal $m$ such that card $A \leqq$ card $R^{\mathrm{m}}=2^{\mathrm{m}}$, so $w w A=n$.) Let $i_{\alpha}: Z_{\alpha} \rightarrow D_{\alpha}$ be one-to-one onto and set $D=\left(\prod_{\alpha} i_{\alpha}\right)\left(D^{\prime}\right)$. Since the projection of $D^{\prime}$ onto any finite subproduct must be onto, it is clear that $D$ is dense, as desired.

\section{REFERENCES}

1. W. Comfort and A. Hager, Dense subspaces of some spaces of continuous functions, Math. Z. 114 (1970), 373-389.

2. E. Hewitt, A remark on density characters, Bull. Amer. Math. Soc. 52 (1946), 641-643. MR 8, 139.

3. E. S. Pondiczery, Power problems in abstract spaces, Duke Math. J. 11 (1944), 835-837. MR 6, 119.

4. V. Saks and R. M. Stephenson, Jr., Products of $\mathfrak{M}$-compact spaces, Proc. Amer. Math. Soc. 28 (1971), 279-288. MR 42 \#8448.

5. Yu. M. Smirnov, On the weight of the ring of bounded continuous functions over a normal space, Mat. Sb. 30(72) (1952), 213-218. (Russian) MR 14, 70.

6. G. Vidossich, Characterizing separability of function spaces, Invent. Math. 10 (1970), 205-208. MR 42 \#2424.

7. S. Willard, General topology, Addison-Wesley, Reading, Mass., 1970. MR 41 \#9173.

Canary Road, Westlake, Oregon 97493 